\title{
Inflammatory myofibroblastic tumor of urinary bladder: case presentation and review of literature
}

\begin{abstract}
Inflammatory myofibroblastic tumor (IMT) is a rare benign spindle cell tumor that has been described in numerous body sites. The diagnosis of this tumor is challenging since it is often misdiagnosed as being malignant even by expert pathologists, which result in patients undergoing unnecessary radical surgery. Here in, we describe the management of a case of IMT of the bladder in a 16year old male patient and review pertinent literature.
\end{abstract}

Keywords: inflammatory myofibroblastic tumor, bladder, partial cystectomy

\author{
Volume I Issue 2 - 2014 \\ Muhammad A Bulbul,' Mohammed Shahait,' \\ Mark Jabbour, ${ }^{2}$ Fouad Boulos, ${ }^{2}$ Yaser El-Hout ${ }^{1}$ \\ 'Department of Surgery,American university of Beirut medical \\ center, Lebanon \\ ${ }^{2}$ Department of Pathology and Laboratory Medicine, American \\ University of Beirut-Medical Center, Lebanon
}

\begin{abstract}
Correspondence: Yaser El-Hout, Department of Surgery, Division of Urology, The American University in Beirut Medical center, PO Box II-0236, Riad Solh II 07 2020, Beirut, Lebanon, Tel: +961331 I 107, Email yelhout@aub.edu
\end{abstract}

Received: November II, 2014 | Published: November 22, 2014
Abbreviations: IMT, inflammatory myofibroblastic tumor; ALK1, anaplastic lymphoma kinase 1; MSA, muscle specific actin; $\mathrm{EBV}$, epstein -barr virus

\section{Introduction}

Most primary bladder tumors in children are predominantly malignant mesenchymal tumors, usually rhabdomyosarcoma. Several uncommon non-urothelial bladder tumors have been described and must be differentiated from sarcomatoid carcinoma. These include postoperative spindle cell nodule, inflammatory myofibroblastic tumor, leiomyoma, hemangioma and neurofibromas. IMT usually affects adolescents. The main presenting symptom is painless gross hematuria. The tumor is usually exophytic on cystoscopy and enhances on CT scan. On pathology, it has heterogeneous microscopic features but invariably has spindle cells.

\section{Case report}

An otherwise healthy 16year old Caucasian male patient was referred with massive painless gross hematuria causing anemia (Hematocrit of 19) that required transfusion with 8 units of blood. He had no history of bleeding disorders, trauma or surgery. Physical examination was essentially negative with rectal examination revealing no masses. Cystoscopy, done elsewhere, was inconclusive due to obscured vision from bleeding. All laboratory tests, including a coagulation profile were normal. Ultrasound showed a $7 \mathrm{~cm}$ mass occupying the right lateral wall of the bladder with no hydronephrosis (Figure 1). Repeat cystoscopy showed a large pedunculated mass arising from the right anterolateral wall with significant bladder clots. Transurethral resection of all visible tumors was performed, submitting around 37 grams of tissue for pathology. Pathology revealed an inflammatory bladder tumor with atypical spindle cells with abundant eosinophilic cytoplasm embedded in a predominantly myxoid stroma, all consistent with IMT; immunohistochemistry showed that tumor was strongly positive to anaplastic lymphoma kinase 1 (ALK1), Cytokeratin AE1/AE3 and local weak Muscle Specific Actin (MSA) (Figure 2). The differential diagnosis included postoperative spindle cell nodule, rhabdomyosarcoma, leiomyoma and sarcomatous carcinoma. The absence of a history of surgical manipulation, dense eosinophilia, severe atypia and the unique immunohistochemical profile argued against the aforementioned diagnoses. Partial cystectomy and right pelvic node dissection was performed. Intra-operative resection margins were negative. The patients did very well post-operatively and a CT scan of the pelvis at one year follow showed no evidence of recurrent tumor.

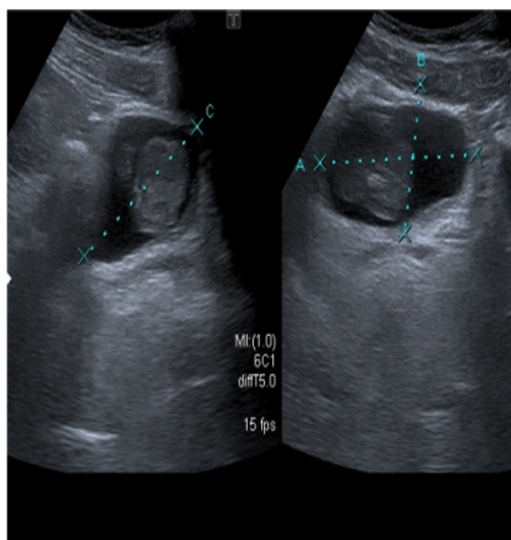

Figure I Ultrasound shows a mass occupying the right lateral wall of the bladder.

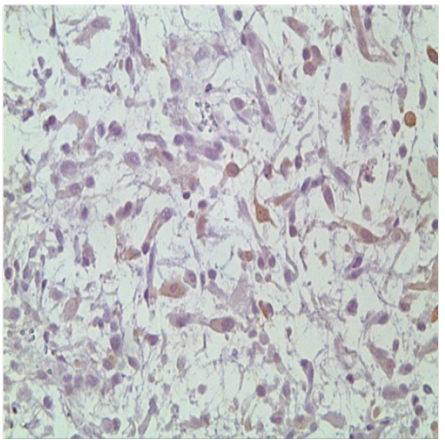

Figure 2 Immunohistochemistry shows that tumor was strongly positive to CKAEI/AE3. 


\section{Discussion}

IMT is a rare spindle cell tumor that has been described in numerous body sites. ${ }^{1,2}$ The World Health Organization classifies IMT as a neoplasm with a tendency for local recurrence and a very low rate of metastasis. ${ }^{3,4}$ In 1939, Brunn described two patients with lung tumors having histological features resembling that of IMT of the genitourinary tract. ${ }^{5}$ IMT of the genitourinary tract was first described by Roth. ${ }^{6}$ It has been described in both children and adults, with an increased predominance in males. ${ }^{7}$ IMT of the bladder occurs mainly in adolescents. Clinical presentation of IMT of the bladder may vary; symptoms may include dysuria, painless hematuria, pelvic pain or symptoms of urinary tract obstruction. ${ }^{8}$ The etiology and pathogenesis of IMT are unknown; many theories have been proposed which include infection by various microbes such as Mycoplasma, Epstein -Barr Virus (EBV), and Human Herpes Virus-8. Altered regulations of cytokine expression, autoimmune etiology, and previous surgery or trauma have also been described as possible etiologies. ${ }^{9-11}$ On cystoscopy, IMT of the bladder is usually exophytic and often polypoid and usually are located in the dome, lateral or posterior walls. ${ }^{12}$ On CT, IMT usually shows heterogeneous enhancement with areas of necrosis.

Magnetic resonance imaging shows an isotense lesion on $\mathrm{T} 1$ weighted images while hypo intense lesions on T2 weighted images. ${ }^{13}$ Most IMTs have heterogeneous microscopic features. Three basic histological findings have been described: 1) loosely arranged spindle cells in edematous myxoid background with an irregular network of small blood vessels and inflammatory cells producing a granulation tissue like appearance; 2) compact spindle cell proliferation with a fascicular or focally storiform pattern mingled with inflammation; and 3) sparsely cellular plate like collagen resembling a scar or desmoid fibromatosis. ${ }^{1}$ The histological finding in our case is compatible with the first histological variation mentioned before. The Immunohistochemistry profile in IMT varies from one case to another, however, most of the tumors stain positive for ALK, SMA, and cytokeratin AE1/AE3 in $65 \%, 71.9 \%$, and $75.3 \%$ of the cases. Chun et al., ${ }^{14}$ found that there is no association between ALK status and outcome after surgery. IMT and bladder sarcoma share to some extent the same immunohistochemical profile as both of them stain positively for vimentin, actin, desmin and keratin to variable degrees. ${ }^{1}$

Partial cystectomy is the mainstay of treatment for IMT. Unfortunately, there is no consensus on the follow-up duration, as there is reported cases in the literature where patient developed local recurrence and metastasis. ${ }^{15}$ There is increasing evidence in the literature that there is no proven role for chemotherapy or radiation therapy. On the other hand, there are reported cases of spontaneous regression of the tumor. ${ }^{16}$ Additionally, Sandhu \& Iacovou ${ }^{17}$ reported a case of an inflammatory bladder tumor invading the rectus sheath which was treated with 4 months of oral antibiotics. They noticed resolution of the tumor at 9months follow up with no local recurrence after 3years.

\section{Conclusion}

Inflammatory bladder tumors are benign and present as a discrete bladder mass. Patients typically present with sterile gross painless hematuria. Astute pathologists should be able to differentiate these tumors from others, especially sarcoma, which is crucial for selecting therapy. Bearing in mind its locally aggressive behavior, open surgery with bladder preservation is the mainstay of treatment.

\section{Acknowledgements}

None.

\section{Conflict of interest}

The author declares no conflict of interest

\section{References}

1. Coffin CM, Watterson J, Priest JR, et al. Extrapulmonary inflammatory myofibroblastic tumor (inflammatory pseudo tumor):A Clinicopathologic and immunohistochemical study of 84 cases. Am J Surg Pathol. 1995;19(8):859-872

2. Chan JK. Inflammatory pseudotumour: a family of lesions of diverse nature and etiologies. Adv Anat Pathol. 1996;3:156-171.

3. Morotti RA, Legman MD, Kerkar N, et al. Pediatric inflammatory myofibroblastic tumor with late metastasis to the lung: case report and review of the literature. Pediatr Dev Pathol. 2005;8(2):224-229.

4. Coffin CM, Fletcher CD. Inflammatory myofibroblastic tumor. In: Fletcher CD, et al. editors. WHO classification of Tumors: Pathology and Genetics tumours of Soft Tissue and Bone. France: IARC Press; 2002. p. 91-93.

5. Brunn H. Two interesting benign lung tumors of contradictory histopathology: remarks on the necessity for maintaining chest tumor registry. J Thorac Surg. 1939;9:119-131.

6. Roth JA. Reactive pseudosarcomatous response in urinary bladder. Urology. 1980;16(6):635-637.

7. Jones EC, Clement PB, Young RH. Inflammatory pseudotumour of the urinary bladder: A clinicopathological, immunohistochemical, ultrastructural, and flow cytometric study of 13 cases. Am J Surg Pathol. 1993;17(3):264-274.

8. Lantz AG, Power NE, Gupta R, et al. inflammatory pseudotumour: A rare cause of hematuria and shock. Urology. 2007;70(2):372.

9. Arber DA, Weiss LM, Chang KL. Detection of Epstein-Barr virus in inflammatory pseudotumour. Semin Diagn Pathol. 1998;15(2):155-160.

10. Gomez-Roman JJ, Ocejo-Vinyals G, Sanchez-Velasco P, et al. Presence of human herpesvirus-8 DNA sequence and over expression of human IL-6 and cyclin D1 in inflammatory myofibroblastic tumor ( inflammatory pseudotumour ). Lab Invest. 2000;80(7):1121-1126.

11. Kojima M, Nakamura S, Itoh $\mathrm{H}$, et al. Inflammatory pseudotumour of the submandibular gland: report of a case presenting with autoimmune disease-like clinical manifestation. Arch Pathol Lab Med. 2001;125(8):1095-1097.

12. Angulo JC, Lopez JI, Flores N. Pseudosarcomatous myofibroblastic proliferation of the bladder: report of 2 cases and literature review. $J$ Urol. 1994;151(4):1008-1012.

13. Kim SH, Yang DM, Kim NR. Polypoid and papillary cystitis mimicking a large transitional carcinoma in a patient without a history of catheterization: Computed tomography and magnetic resonance findings. J Comput Assist Tomogr. 2004;28(4):485-487.

14. Chun J, Teoh, Chan N, et al. Inflammatory Myofibroblastic Tumors of the Urinary Bladder: A Systematic Review. J Urol. 2014;84(3):503-508.

15. Kim HW, Choi YH, Kang SM, et al. Malignant inflammatory myofibroblastic tumor of the bladder with rapid progression. Korean $J$ Urol. 2012;53(9):657-661.

16. Sutphin M, Middleton AW. Eosinophilic cystitis in children: a selflimited process. J Urol. 1984;132(1):117-119.

17. Sandhu SS, Iacovou JW. Pseudotumour of the bladder. $J$ R Soc Med. 1997;90:46-47. 